\title{
Dynamic Contrast-enhanced Magnetic Resonance Imaging in Diagnosis of Cavernous Hemangioma of Cavernous Sinus
}

Sir,

Cavernous hemangiomas of the cavernous sinus (CHCS) comprise $<1 \%$ of all parasellar masses and closely mimic tumors such as meningioma, pituitary macroadenoma, or schwannomas at this site. ${ }^{[1]}$ Although magnetic resonance imaging (MRI) can help in diagnosis, addition of dynamic contrast-enhanced sequence to conventional MR sequences demonstrates the characteristic gradual "filling in" of the lesion and helps in preoperative diagnosis with a better accuracy, thus guiding appropriate surgical approach and reducing the associated perioperative morbidity.

A 43-year-old female presented with on and off headache and diminution of vision for 2 months. She had left-sided facial pain for 2 months. X-ray of the skull lateral view revealed widened sella. MRI showed T1 hypointense and T2 hyperintense lesion in the suprasellar and left parasellar location, encasing the left cavernous internal carotid artery (ICA) completely without stenosis. Dynamic postcontrast MRI was performed after giving a bolus dose of $10 \mathrm{ml}$ of gadolinium contrast agent at a rate of $3 \mathrm{ml}$ per second. Dynamic images were acquired in six phases (at TR/TE-560/10) which show progressive filling in of the lesion [Figure 1]. Digital subtraction angiography had also demonstrated tumor blush. Based on characteristic imaging findings, a diagnosis of CHCS was made. Surgery was not preferred in this patient as the lesion was completely encasing left cavernous ICA and in relation with right cavernous sinus. The patient underwent radiotherapy. Follow-up imaging 4 months after radiotherapy showed significant reduction in the size of the lesion [Figure 2]. The patient also improved symptomatically.

Although extra-axial cavernous hemangiomas have the same histologic features as intra-axial lesions, former have different clinical pictures, natural history, and radiologic findings. CHCS present with slowly progressive symptoms due to compression on adjacent cranial nerves and retro-orbital structures. Other symptoms include headache, proptosis, and hypopituitarism. They are frequently encountered in the fourth and fifth decades of life and have a female predominance and tendency to proliferate during pregnancy. Although highly vascular, CHCS do not manifest with acute spontaneous hemorrhage. ${ }^{[2]}$

Closest differential of CHCS includes suprasellar meningiomas, pituitary macroadenoma, and schwannoma. Meningiomas are usually isointense to gray matter on $\mathrm{T} 1$ and $\mathrm{T} 2$ sequences and show intense homogeneous postcontrast enhancement. A dural tail is frequently seen extending away from the edge of the tumor. Meningiomas narrow the lumen of adjacent ICA. Pituitary macroadenomas are heterogeneous lesions usually hypo- to iso-intense on $\mathrm{T} 1$ and mildly hyperintense on $\mathrm{T} 2$. Pituitary adenomas usually do not narrow the lumen of ICA. Schwannomas are isointense to hypointense masses on T1 images, mostly $\mathrm{T} 2$ hyperintense, and show heterogeneous contrast enhancement. A clue to the diagnosis is that they follow the expected course of the nerves from which they arise. ${ }^{[3]}$

CHCS grow as asymmetrical dumbbell-shaped masses occupying the sella, suprasellar, and parasellar region causing mass effect and encasement of neurovascular structure. Characteristic finding is encasement of vessels without occlusion. These lesions are isointense to hypointense on T1-weighted imagec (T1-WI) and markedly hyperintense on T2-WI. These highly vascular lesions show intense homogeneous postcontrast enhancement. ${ }^{[4]}$ This intense enhancement is attributed to large amount of thin-walled vascular sinusoids with varying amount of intervening connective tissue according to the type of CHCS. Actually, in early phases, heterogeneous enhancement is noted, then it gradually become homogeneous as reported by Jinhu et al. in their sequential axial, coronal, and sagittal 

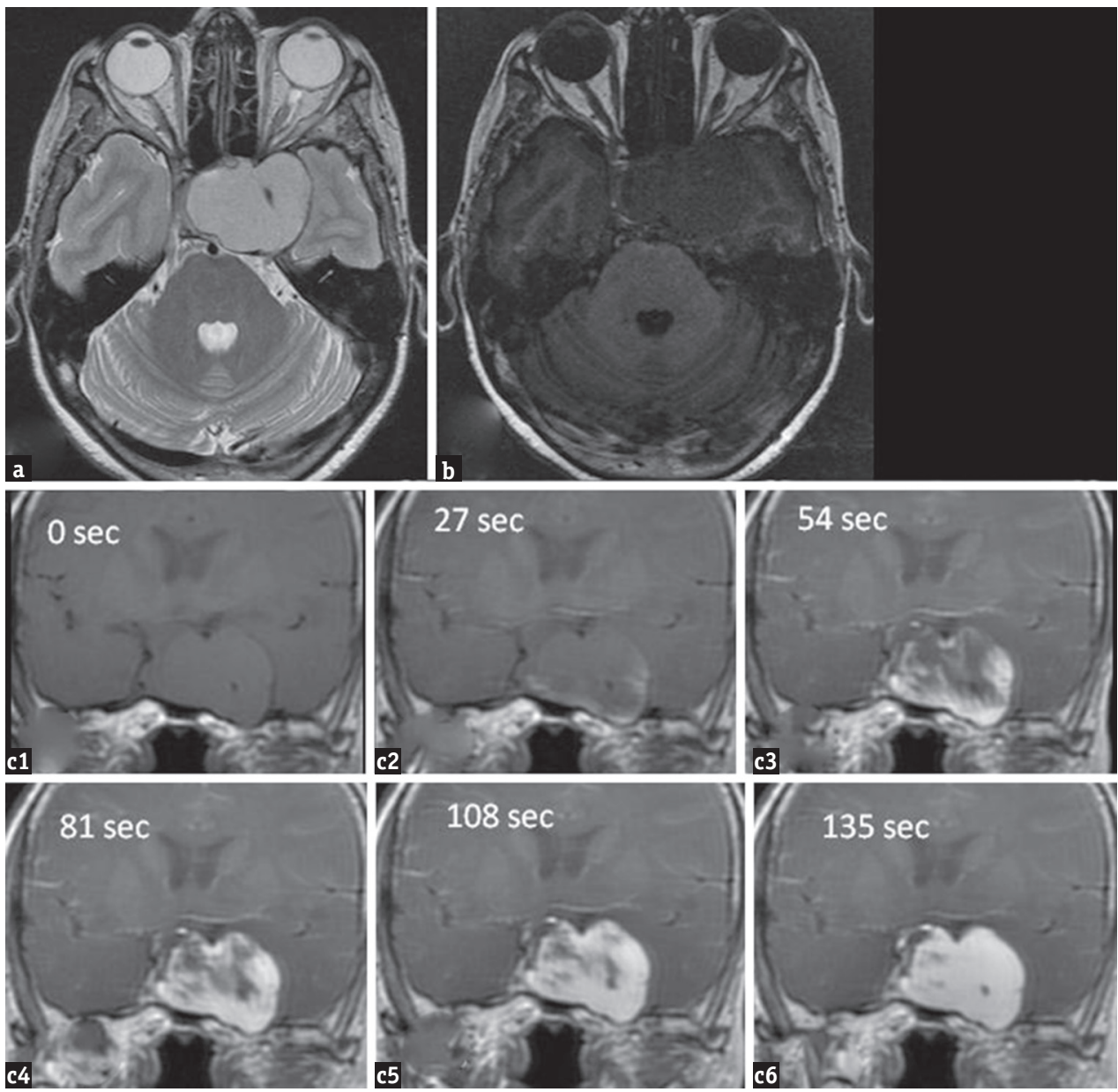

Figure 1: (a) Axial T2-weighted magnetic resonance imaging showing well-defined homogeneously hyperintense lesion in the suprasellar and left parasellar region with encasement of the left cavernous internal carotid artery. The lesion appears mildly hypointense on T1-weighted image (b). Dynamic contrast-enhanced imaging in coronal plane obtained in 6 phases at an interval of $27 \mathrm{~s}$ showing progressive filling in of the lesion and intense enhancement (c1-c6)

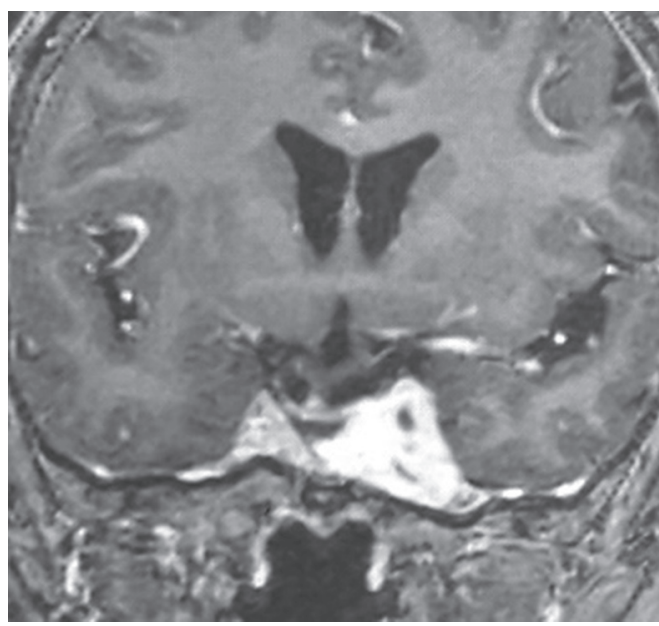

Figure 2: Follow-up imaging 4 months after radiotherapy. Postcontrast coronal T1-weighted image showing significant reduction in size of the lesion

images. ${ }^{[1]}$ Our dynamic contrast-enhanced sequence demonstrates the characteristic gradual filling in of the lesion on successive phases. Progressive contrast "filling in" in the parasellar and suprasellar lesion on dynamic contrast-enhanced
MR images can suggest the preoperative diagnosis of CHCS and reduce operative morbidity as these lesions have high propensity to bleed during surgery. Alternative treatment modalities include gamma knife radiosurgery and radiotherapy.

Progressive contrast filling in a T2 brightly hyperintense and $\mathrm{T} 1$ hypointense parasellar mass lesion involving the cavernous sinus is highly suggestive of CHCS.

\section{Acknowledgment}

We thank Department of Neurosurgery and Radiotherapy for the patient management.

\section{Financial support and sponsorship Nil.}

\section{Conflicts of interest}

There are no conflicts of interest.

Suprava Naik, Rajendra V. Phadke ${ }^{1}$, Arpit Taunk ${ }^{1}$, Vivek Singh ${ }^{1}$, Sanjeev Kumar Bhoi ${ }^{2}$

Departments of Radiodiagnosis and ${ }^{2}$ Neurology, All India Institute of Medical Sciences, Bhubaneswar, Odisha, ${ }^{1}$ Department of Radiodiagnosis, Sanjay Gandhi Postgraduate Institute of Medical Sciences, Lucknow, Uttar Pradesh, India 
Address for correspondence: Dr. Suprava Naik, Department of Radiodiagnosis, All India Institute of Medical Sciences, Bhubaneswar, Odisha, India.

E-mail: drsuprava.rd@gmail.com

\section{REFERENCES}

1. Jinhu Y, Jianping D, Xin L, Yuanli Z. Dynamic enhancement features of cavernous sinus cavernous hemangiomas on conventional contrast-enhanced MR imaging. AJNR Am J Neuroradiol 2008;29:577-81.

2. Salanitri GC, Stuckey SL, Murphy M. Extracerebral cavernous hemangioma of the cavernous sinus: Diagnosis with MR imaging and labeled red cell blood pool scintigraphy. AJNR Am J Neuroradiol 2004;25:280-4.

3. Razek AA, Castillo M. Imaging lesions of the cavernous sinus. AJNR Am J Neuroradiol 2009;30:444-52.

4. Yadav RR, Boruah DK, Yadav G, Pandey R, Phadke RV. Imaging characteristics of cavernous sinus cavernous hemangiomas. Neuroradiol J 2012;25:515-24.
This is an open access article distributed under the terms of the Creative Commons Attribution-NonCommercial-ShareAlike 3.0 License, which allows others to remix, tweak, and build upon the work non-commercially, as long as the author is credited and the new creations are licensed under the identical terms.

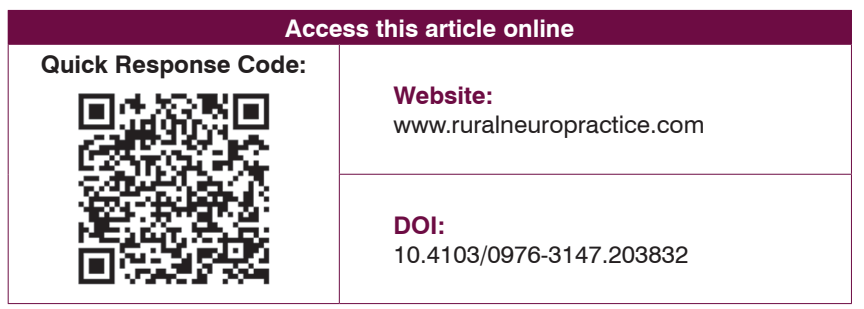

How to cite this article: Naik S, Phadke RV, Taunk A, Singh V, Bhoi SK. Dynamic contrast-enhanced magnetic resonance imaging in diagnosis of cavernous hemangioma of cavernous sinus. J Neurosci Rural Pract 2017;8:311-3.

(c) 2017 Journal of Neurosciences in Rural Practice | Published by Wolters Kluwer - Medknow 